\title{
Validation of the Electromagnetic Physical Processes with Software SPENVIS
}

\author{
Filomena Loffredo $^{1,2, \text { *, Mariagabriella Pugliese }}{ }^{1,2}$, Maria Quarto ${ }^{1,2}$, Vincenzo Roca ${ }^{1,2}$, \\ Valerio Pisacane $^{3}$, Renato Aurigemma ${ }^{4}$ \\ ${ }^{1}$ Dipartimento di Fisica, Università di Napoli Federico II, Naples, Italy \\ ${ }^{2}$ Istituto Nazionale di Fisica Nucleare (INFN), Sez. Napoli, Naples, Italy \\ ${ }^{3}$ Euro.Soft srl, Naples, Italy \\ ${ }^{4}$ Consorzio Società Aerospaziale Mediterranea (SAM), Naples, Italy
}

Email address:

floffredo@na.infn.it (F. Loffredo)

\section{To cite this article:}

Filomena Loffredo, Mariagabriella Pugliese, Maria Quarto, Vincenzo Roca, Valerio Pisacane, Renato Aurigemma. Validation of the Electromagnetic Physical Processes with Software SPENVIS. American Journal of Environmental Protection.

Vol. 4, No. 6, 2015, pp. 275-278. doi: 10.11648/j.ajep.20150406.12

\begin{abstract}
The Space Radiation represents a serious risk for astronauts during space missions. The risk related to the space radiation exposure could involve acute and/or late effects. The Solar Cosmic Radiation that consists of protons $(\approx 98 \%)$ with a very wide spectrum in energy (up to several $\mathrm{GeV}$ ), is the major source of exposure for the crew. In this paper we present the results of the validation of the electromagnetic physical processes with the aim to contribute to the study of radiation protection for astronauts, in particular against the radiation due to the Solar Particle Events (SPE). The simulation was performed using MULASSIS, a module to the software SPENVIS, with protons as source, in the energy range from $800 \mathrm{MeV}$ to $1.2 \mathrm{GeV}$, on a slab of aluminum of mass thickness of $20 \mathrm{~g} / \mathrm{cm}^{2}$. The results obtained by the simulation were compared with PSTAR database of the NIST. Finally, a comparison between SPENVIS and Geant4-9.6p2 was performed.
\end{abstract}

Keywords: Space Radiation, SPENVIS-MULASSIS, Geant4, Protons, Al Target

\section{Introduction}

During space missions it is important to consider that the astronauts are subjected to risks from exposure to radiation. On the Earth, three parameters can be considered to limit the exposure to the radiation, exposure time, distance between source and target and shielding. Instead, the radiation protection in the space is a very complex problem because the only possibility to provide it is the shielding. It should have important features: first of all, it has to be light, because very heavy shields are impractical on spaceships and especially must take into account the interaction of radiation with the materials, which produce secondary radiation that can be very harmful to the health of astronauts. The space radiation consist of a mixed field of radiations where, it is possible to distinguish three categories: i) Van Allen radiation belts, ii) Galactic Cosmic Radiation (GCR) and iii) Solar Particle Events (SPE) [4]. In particular the SPE, composed for $98 \%$ of protons with energies up to several $\mathrm{GeV}$ and high fluences $\left(\approx 10^{11}\right.$ particles $\left./ \mathrm{cm}^{2}\right)$, represent one of the main health risk for long duration manned interplanetary missions [8]. The study of the behavior of protons at energies of about $1 \mathrm{GeV}$ seems to be very interesting because the main contribution to the equivalent dose is provided by them and the remaining is attributed to heavy ions $(10 \%)$. The Solar Particles Events show a maximum intensity during maximum solar intensity, they are seldom, but their occurrence is not negligible since represent the greatest danger to the unshielded crew. Intense SPE can be life threatening or induce acute radiation syndromes (such as nausea and possibly vomiting). Previous studies showed that for the SPE, the level of shielding for the most dangerous events, needed is established to be about $20 \mathrm{~g} / \mathrm{cm}^{2}$, typical mass thickness of the storm shelters [4].

For the health risk assessment transport codes are used, but these have a high degree of uncertainty, due to the fact that the experimental data available for the validation of different calculation models are still few. Since the limits of career recommended for activities in Low Earth Orbit (LEO) by the National Council on Radiation Protection and Measurements 
(NCRP) are estimated by combining data from personal dosimeters of astronauts with the results obtained using the calculation models, it is clear the importance of implementing these using all the information from research conducted both in flight, aboard the International Space Station (ISS), at accelerators dedicated (at the NASA Space Radiation Laboratory at Brookhaven and the National Institute of Radiological Sciences in Chiba).

In this paper, by using MULASSIS, the validation of the electromagnetic physical processes was performed in a range of energy characteristic of SPE. There are other codes, but are not widely available, such as HZETRN which is an analytic code, have shown a good performance [10].

The simulation was performed assuming a slab of aluminum of $20 \mathrm{~g} / \mathrm{cm}^{2}$ as shielding, whose is established to be the level of shielding for the most dangerous events. The primary beam consists of protons of energy varying between 800 to $1200 \mathrm{MeV}$. The results of the electromagnetic Stopping Power obtained with MULASSIS and Geant4$9.6 \mathrm{p} 2$, for different values of energy, and the comparison with the NIST data are reported.

\section{Materials and Methods}

\subsection{SPENVIS - MULASSIS}

SPENVIS is an interactive tool developed by the European Space Agency (ESA) and it provides information on some space parameters, such as orbit, total mission duration, solar radiation pressure, perigee and apogee altitude etc. [5]. It has also been made available through the SPENVIS web-site (http://www.spenvis.oma.be/spenvis/). SPENVIS consists of an integrated set of models that facilitates its use in the space environment. The concept of project is the core of SPENVIS. A project is a set of input and output data obtained from a series of related runs.

MULASSIS is one of the radiation analysis tools derived from ESA's investment in the Geant4 Collaboration, in fact it is a module of SPENVIS based on the use of the toolkit Geant4 (Geant4-9.2p1), for study and simulation of the effects of radiation on the shielding in space environment [7, 9]. MULASSIS tool is a significant improvement over conventional engineering tools for simulating shielding effects such as when interact with the radiation. Following, its main capabilities are shown:

- One-dimensional simulation of shielding effects: Multiple layers of predefined or user-defined materials e.g. geometry definition of the target, the number of layers that compose of each thickness, the type and the size of materials in the NIST database (however if the material is not in this database, the software allows to "create" it specifying the name, chemical formula and density).

- General source particle distribution: Standard spectral distribution or pointwise fit; Unidirectional / isotropic / cosine-law angular distribution; Spectra output by other SPENVIS utilities [7].
The physical models that can be implemented in SPENVIS for protons are reported in Table 1:

Tab. 1. The physical models, for protons, that can be implemented in SPENVIS.

\begin{tabular}{ll}
\hline Energy Range & Physical models \\
\hline$<10 \mathrm{GeV}$ & Standard EM or Low-Energy EM - G4Binary \\
$>10 \mathrm{GeV}$ & Above + G4QGSM \\
\hline
\end{tabular}

In the output of SPENVIS it is possible to choose among four types of the analysis: 1) Fluence analysis, 2) Nonionizing dose, 3) Energy deposition and total ionizing dose and 4) Pulse-height spectrum analysis. To use MULASSIS, no programming in $\mathrm{C}++$ is required, thus, removing one of the main obstacles for Monte-Carlo simulation becoming a mainstream engineering tool in space radiation analysis [7].

\subsection{GEANT4}

Geant 4 is a versatile and powerful toolkit to simulate the passage of particles through matter. It includes a large variety of physics functionality for each particle type in the energy range from a few $\mathrm{eV}$ to several $\mathrm{TeV}$ [6]. It is possible to construct a detailed geometry that reproduces the irradiation conditions. Geant 4 allows users to choose among a wide range of models, driven by theory or data, or based on parameterisations to describe the different physical phenomena depending on particle type and energy range.

With respect to other general-purpose Monte Carlo systems for particle transport, Geant 4 is characterized by the peculiarity of encompassing a very large variety of physics modeling options. Users are responsible for selecting the physics configuration of their experimental applications in fact the nature of Geant 4 as a toolkit prevents the definition of any "default" physics configuration. The task of optimizing the physics configuration for a given experimental scenario is guided by the body of knowledge of Geant4 physics validation available in the literature [1], which encompasses both validation tests of the fundamental components of Geant 4 models (e.g. cross sections, stopping powers, secondary particle production etc.) and comparisons of complex simulated observables (e.g. energy deposition patterns) with experimental measurements.

\subsection{Experimental Setup}

The experimental setup adopted for the validation of the electromagnetic physical processes, is reported in Fig. 1. The simulated geometry is constituted by a point source of protons with energies from $800 \mathrm{MeV}$ to $1200 \mathrm{MeV}$, in the vacuum. This energy range is typical of the protons that constitute the solar particle events. The target is a slab of aluminum $\left(\rho \approx 2.7 \mathrm{~g} / \mathrm{cm}^{3}\right)$, in the shape of a parallelepiped, of a mass thickness $20 \mathrm{~g} / \mathrm{cm}^{2}$ in the direction of incidence of the primary beam $(\mathrm{z}=7.4 \mathrm{~cm})$ and with surfaces $30 \times 30 \mathrm{~cm}$. To compare the values of the electronic stopping power it is necessary that the energy of the primary beam does not vary appreciably in the target, condition assured by a small thickness. In this configuration, the thickness used aluminum 
is $5 \%$ of the projected-range of the protons, which varies from $300 \mathrm{~g} / \mathrm{cm}^{2}$ to $530 \mathrm{~g} / \mathrm{cm}^{2}$ in the energy range from 800 $\mathrm{MeV}$ to $1.2 \mathrm{GeV}$ in referring to the tables in the database PSTAR [3].

The physical model considered for the study of the electromagnetic physical processes is Low-Energy EM (Multiple scattering, Ionization and production of $\delta$ rays), as reported in Table 1. The latter take into account the different features that are relevant to several experimental domains, such as space science. The validation of the electromagnetic physical process was essential to assess the energy loss by ionization and excitation of the protons in aluminum.

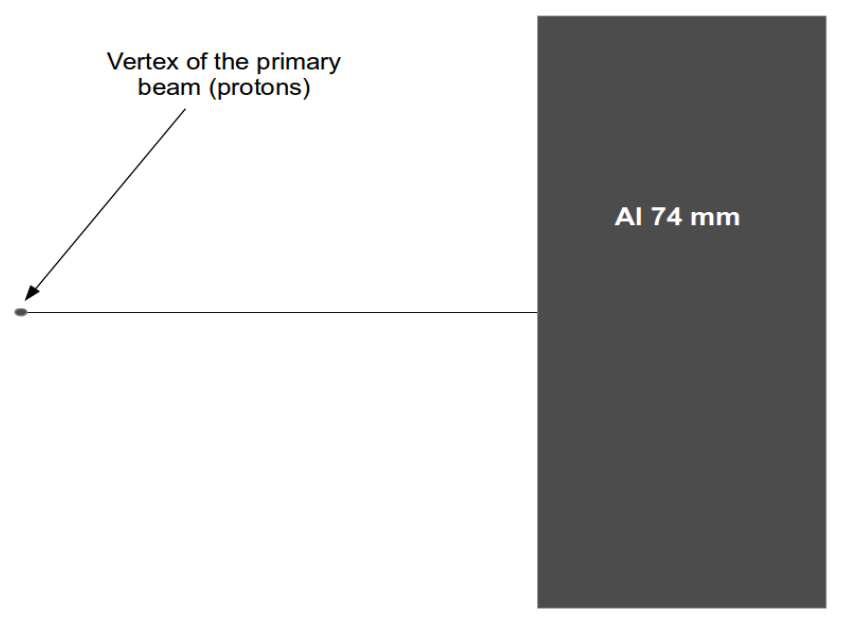

Fig. 1. Experimental Setup: target is a slab of aluminum $\left(\rho \approx 2.7 \mathrm{~g} / \mathrm{cm}^{3}\right)$ of a thickness $74 \mathrm{~mm}$, in the direction of incidence of the primary beam.

\section{Results}

The values of the electronic stopping power obtained using the codes, with relative uncertainty, and those calculated by PSTAR reported in the table 2.

The results reported in tab. 2 show the expected trend of stopping power, which decreases with increasing energy. With the use of the software SPENVIS-MULASSIS, the stopping power and its relative uncertainty, were obtained from the output 'Energy deposition' that was normalized respect to the mass thickness. The values shown in the PSTAR database of the NIST are supplied without error because the tables of stopping power and range of protons are calculated in accordance with the Bethe Bloch formula. The Stopping Power of the protons in the energy range of interest, in the aluminum slab are reported in fig. 2.

Tab. 2. The comparison, of the stopping power of protons in aluminum, SPENVIS-MULASSIS, Geant4-9.6p2 and PSTAR of the NIST.

\begin{tabular}{|c|c|c|c|}
\hline $\begin{array}{l}\text { Energy } \\
(\mathrm{MeV})\end{array}$ & $\begin{array}{l}\text { Stopping Power } \\
\text { SPENVIS-MULASSIS } \\
\mathrm{MeV} \mathrm{cm}^{2} / \mathrm{g}\end{array}$ & 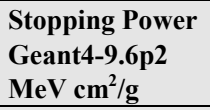 & $\begin{array}{l}\text { Stopping Power } \\
\text { PSTAR- NIST } \\
\mathrm{MeV} \mathrm{cm}^{2} / \mathrm{g} \\
\end{array}$ \\
\hline 800 & $1.89 \pm 0.05$ & $1.87 \pm 0.20$ & 1.85 \\
\hline 850 & $1.85 \pm 0.05$ & $1.86 \pm 0.16$ & 1.82 \\
\hline 900 & $1.83 \pm 0.05$ & $1.79 \pm 0.14$ & 1.79 \\
\hline 950 & $1.78 \pm 0.05$ & $1.79 \pm 0.19$ & 1.77 \\
\hline 1000 & $1.78 \pm 0.05$ & $1.74 \pm 0.16$ & 1.75 \\
\hline 1050 & $1.77 \pm 0.05$ & $1.75 \pm 0.29$ & 1.73 \\
\hline 1100 & $1.74 \pm 0.05$ & $1.70 \pm 0.26$ & 1.72 \\
\hline 1150 & $1.69 \pm 0.05$ & $1.72 \pm 0.16$ & 1.71 \\
\hline 1200 & $1.65 \pm 0.05$ & $1.70 \pm 0.12$ & 1.69 \\
\hline
\end{tabular}

\section{Stopping Power in Al Target}

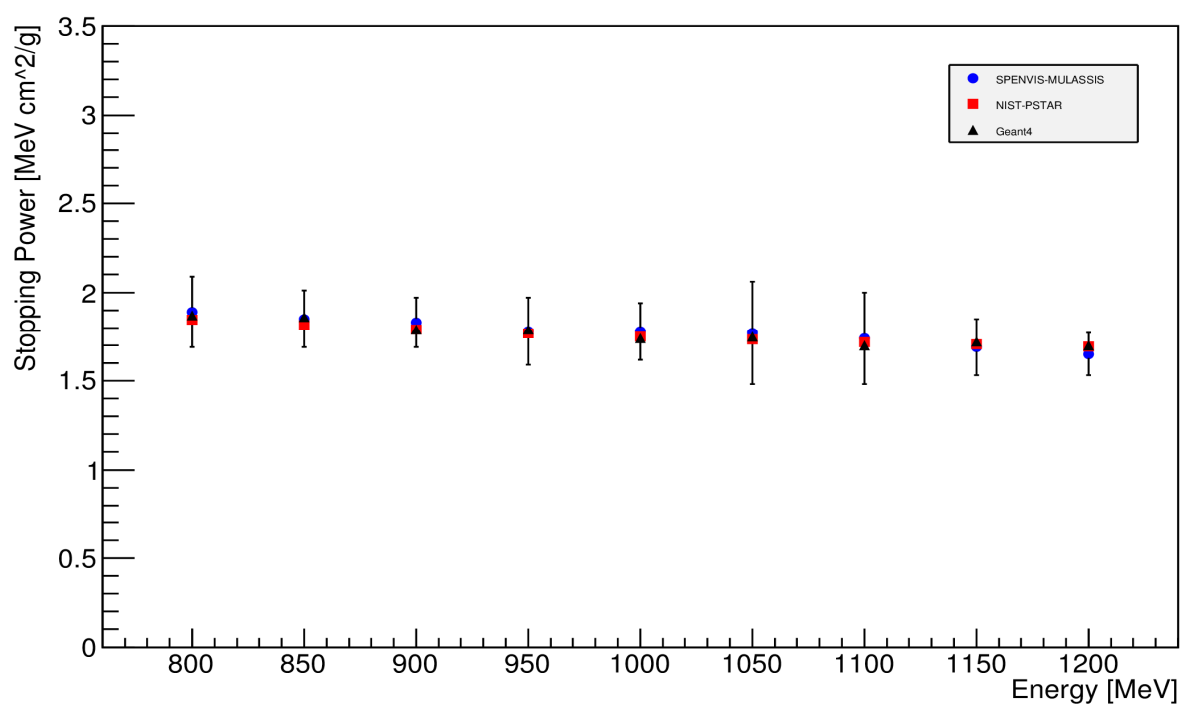

Fig. 2. Comparison of the values of the electronic stopping power between SPENVIS-MULASSIS software, Geant4-9.6p2 and database PSTAR, of protons in aluminum in the energy range from $800 \mathrm{MeV}$ to $1.2 \mathrm{GeV}$.

The blue circles represent the Stopping Power values obtained with the MULASSIS module, the red squares represent the values shown in the PSTAR database and the black triangles represent the values obtained with the Geant4 toolkit. The table and graph show that there is a good agreement between simulated data, with SPENVIS- 
MULASSIS and Geant4, and the tabulated data PSTARNIST because the tabulated value is in the range determined by the standard deviation.

\section{Conclusions}

The agreement of SPENVIS-MULASSIS and Geant4 stopping powers is not surprising since the first code relies on the second for describing particle interactions with matter. According to the Geant 4 release notes, the relevant Geant4 model implementations did not change between the Geant 4 version used in MULASSIS (9.2 p1) compared to the standalone Geant 4 version adopted in the paper (9.6 p2). Hence, the comparison between SPENVIS-MULASSIS and Geant4 reduces to simple verification if Geant4 is correctly used within SPENVIS-MULASSIS. From a theoretical point of view it can be well argued why the difference between Geant 4 and NIST-PSTAR stopping powers should be small for protons in Aluminium at these energies. NIST-PSTAR reproduces tables from the ICRU49 report, which, like Geant4, relies on the relativistic Bethe formula at higher energies (i.e. above a few MeV). The only "free" parameter in the Bethe formula is the mean ionization potential, for which Geant4 uses by default NIST values, i.e. the same values as adopted in NISTPSTAR (166 eV for Aluminium).

In addition to the Bethe formula, ICRU49 and Geant 4 both apply higher order correction terms, which include the density effect correction, the Bloch correction, the Barkas correction, as well as shell corrections. For the proton energies discussed in the paper, the largest correction term comes from the density effect, which however contributes still less than $1 \%$ to the Bethe term; all other terms contribute less than $0.1 \%$ [11]. This means that the expected difference between Geant 4 and ICRU49 due to higher order corrections can be expected to be very small, even if Geant4 and ICRU49 would rely on different models for describing the density effect.

Apart from theoretical grounds, one could argue that differences may arise from code details. For example, Geant 4 pre-computes many physics quantities in form of tables which are then interpolated during the simulation run. However, stopping powers in this energy regime exhibit only a moderate variation with energy, hence the error due to interpolations is likely small and is probably smaller than the statistical error of the results reported in the paper.

Last but not least, the stopping power models in Geant4 have been verified previously against NIST-PSTAR data [2], in this reference shows a direct Geant4-PSTAR stopping power comparison for Aluminium over a wide energy range, demonstrating a good agreement between Geant4 and the NIST tabulation.

\section{References}

[1] S. Agostinelli, J. Allison, K. Amako, J. Apostolakis, et al., Geant4 - A simulation toolkit. Nucl. Instrum, Meth. Phys. Res. A506 (3), 250-303, 2003.

[2] K. Amako, S. Guatelli, V. N. Ivanchenko, M. Maire, B. Mascialino, K. Murakami, P. Nieminen, L. Pandola, S. Parlati, M. G. Pia, M. Piergentili, T. Sasaki, and L. Urban, Comparison of Geant4 Electromagnetic Physics Models Against the NIST Reference Data, IEEE Trans. Nuc. Sci, Vol $52(2005)$.

[3] M. J. Berger, ESTAR, PSTAR, and ASTAR: Computer Programs for Calculating Stopping-Power and Range Tables for Electrons, Protons, and Helium Ions (version 1.2.3), National Institute of Standards and Technology, Gaithersburg, MD., [Online] 2005. Available: http://physics.nist.gov/Star.

[4] M. Durante, F.A. Cucinotta, Physical basis of radiation protection in space travel. Rev. Mod. Phy. 83, 1245, 2011.

[5] D. Heynderickx, B. Quaghebeur, J. Wera, E. J. Daly, H. D. R. Evans, New radiation environment and effects models in ESA's space environment information system (SPENVIS) . Proceedings of the 7th European Conference on Radiation and Its Effects on Components and Systems, RADECS 2003.

[6] A. Ivantchenko, P. Nieminen, S. Incerti, G. Santin, V. Ivantchenko, V. Grichine, J. Allison, 40th COSPAR Scientific Assembly. Held 2-10 August 2014, in Moscow, Russia, Abstract F2.2-38-14, 2014.

[7] F. Lei, P. R. Truscott, C. S. Dyer, B. Quaghebeur, D. Heynderickx, P. Nieminen, H. Evans, E. Daly, MULASSIS: A Geant4-Based Multilayered Shielding Simulation Tool, IEEE Trans. Nucl. Sci. 49 (6), 2788-2793, 2003.

[8] R. E. McGuire, T. T. von Rosenvinge, F. B. McDonald, The composition of solar energetic particles, Astrophysical Journal 301, 938-961, 1986.

[9] P. R. Truscott, F. Lei, C. Dyer, B. Quaghebeur, D. Heyndericks, P. Nieminen, H. Evans, E. Daly, A. Mohammadzadeh, G. Hopkinson, MULASSIS - Monte Carlo Radiation Shielding Simulation for Space Applications Made Easy, European Space Components Information Exchange System, ESCIES, 2003.

[10] J. W. Wilson, F. F. Badavi, F. A. Cucinotta, J. L. Shinn, G. D. Badhhwar, R. Silberberg, C.H. Tsao, L. W. Townsend, R. K. Tripathi, HZETRN: Description of a Free-Space Ion and Nucleon Transport and Shielding Computer Program, NASA TP-3495, 1995.

[11] J. F. Ziegler, The Stopping of Energetic Light Ions in Elemental Matter HZETRN: Description of a Free-Space Ion and Nucleon Transport and Shielding Computer Program, NASA TP-3495, 1995. 